\title{
Detection of serum IgM and IgG for COVID-19 diagnosis
}

\author{
Ling Zhong ${ }^{1,2,3 \dagger}$, Junlan Chuan ${ }^{1,2 \dagger}$, Bo Gong ${ }^{1,2,3 \dagger}$, Ping Shuai ${ }^{4}$, Yu Zhou ${ }^{1,2,3}$, Yi Zhang ${ }^{1,2,3}$, \\ Zhilin Jiang ${ }^{1,2,3}$, Dingding Zhang ${ }^{1,2,3}$, Xiaoqi Liu ${ }^{1,2,3}$, Shi Ma ${ }^{1,2,3}$, Yi Huang ${ }^{1}$, He Lin ${ }^{1,2,3}$, \\ Qingwei Wang ${ }^{1,2,3}$, Lulin Huang ${ }^{1,2,3}$, Dan Jiang ${ }^{1,2,3}$, Fang Hao ${ }^{1,2,3}$, Juan Tang ${ }^{5}$, Chunqi Zheng ${ }^{5}$, \\ Hua $\mathrm{Yu}^{1}$, Zhibin Wang ${ }^{1}$, Qi Jiang ${ }^{6}$, Tao Zeng ${ }^{7}$, Mei Luo ${ }^{7}$, Fanwei Zeng ${ }^{8}$, Fanxin Zeng, \\ Jianghai Liu ${ }^{9}$, Junxi Tian ${ }^{10}$, Yu Xu ${ }^{10}$, Tengxiang Long ${ }^{10}$, Kaiju Xu ${ }^{11}$, Xingxiang Yang ${ }^{11}$, \\ Yuping Liu ${ }^{4}$, Yi Shi ${ }^{1,2,3^{*}}$, Li Jiang ${ }^{1,2,3^{*}}$ \& Zhenglin Yang ${ }^{1,2,3^{*}}$ \\ ${ }^{1}$ The Key Laboratory for Human Disease Gene Study of Sichuan Province and Institute of Laboratory Medicine, Sichuan Provincial People's \\ Hospital, University of Electronic Science and Technology of China, Chengdu 610072, China; \\ ${ }^{2}$ Research Unit for Blindness Prevention of Chinese Academy of Medical Sciences (2019RU026), Sichuan Academy of Medical Sciences, \\ Chengdu 610072, China; \\ ${ }^{3}$ Natural Products Research Center, Institute of Chengdu Biology, Sichuan Translational Medicine Hospital, Chinese Academy of Sciences, \\ Chengdu 610072, China; \\ ${ }^{4}$ Health Management Center, Sichuan Academy of Medical Sciences \& Sichuan Provincial People's Hospital, University of Electronic Science \\ and Technology of China, Chengdu 610072, China; \\ ${ }^{5}$ Infectious Disease Department, Zigong first people's Hospital, Zigong 643000, China; \\ ${ }^{6}$ Sichuan Mianyang 404 Hospital, Mianyang 621000, China; \\ ${ }^{7}$ Infectious Diseases Laboratory, Public Health and Clinical Center of Chengdu, Chengdu 610041, China; \\ ${ }^{8}$ Sichuan Dazhou Central Hosptical, Dazhou 635000, China; \\ ${ }^{9}$ ABLINK Biotech, Chengdu 610041, China; \\ ${ }^{10}$ Maccura Biotechnology Co., Ltd., Chengdu 610041, China; \\ ${ }^{11}$ Infectious Disease Department, Sichuan Provincial People's Hospital, University of Electronic Science and Technology of China, Chengdu \\ 610041, China
}

Received March 3, 2020; accepted March 23, 2020; published online March 25, 2020

Citation: Zhong, L., Chuan, J., Gong, B., Shuai, P., Zhou, Y., Zhang, Y., Jiang, Z., Zhang, D., Liu, X., Ma, S. et al. (2020). Detection of serum IgM and IgG for COVID-19 diagnosis. Sci China Life Sci 63, 777-780. https://doi.org/10.1007/s11427-020-1688-9

Dear Editor,

Infection with the novel coronavirus (SARS-CoV-2, which is the virus responsible for the coronavirus disease 2019 (COVID-19)) was first reported in Wuhan, China on December 31, 2019. The outbreak of COVID-19 remains ongoing and was linked to more than 80,000 infected patients and more than 3,000 deaths in China as of March 7, 2020

$\uparrow$ Contributed equally to this work

*Corresponding authors (Zhenglin Yang, email: zliny@yahoo.com; Jiang Li, email: jiang.li@hsc.utah.edu; Yi Shi, email: shiyi1614@126.com)
(Holshue et al., 2020).

Currently, the real-time RT-PCR assay is the gold-standard methodto diagnose SARS-CoV-2. However, false-negative cases have been reported due to problems with sample collection and transportation, RNA extraction, enzyme inhibitors, and the RT-PCR method (Yang et al., 2020; Lu et al., 2020). By contrast, conventional serological assays, such as the enzyme-linked immunoassay (ELISA) for specific IgM and IgG antibodies, have a high-throughput advantage, and they avoid false -negative cases that occur with the RTPCR method (Xiao et al., 2020).

To assay the concentration of antibodies ( $\operatorname{IgM}$ and $\mathrm{IgG}$ ) 
responsible for the coronavirus spike (S) and nucleocapsid (N) proteins in the serum, we developed ELISA and chemiluminescence methods for serum samples to detect IgM and $\mathrm{IgG}$ antibodies responsible forS and $\mathrm{N}$ proteins, respectively. The $\mathrm{N}$ gene of the SARS-CoV-2 was cloned into a pET28a vector, and the $\mathrm{S}$ gene was cloned into a pMFcIg vector. The proteins were expressed in E. coli and FreeStyle 293-F cells and purified by affinity chromatography. Both immunological methods were compared to the nucleic acid detection assay. Sample size calculation was based on the desired sensitivity and specificity (Negida et al., 2019) and the operator received curve (ROC) was constructed with SPSS version 25.0.

The $\mathrm{N}$ proteins appeared as an obvious band at approximately $50 \mathrm{kD}$, which is consistent with their theoretical molecular weight of $49.1 \mathrm{kD}$ (Figure S1A and S1C). The the RBD-mFc band was consistent with its estimated molecular weight of approximately $60 \mathrm{kD}$ (Figure S1B). Because there were several glycosylation sites, with most located in the S1 subunit of the $\mathrm{S}$ protein of the coronavirus, the RBD-mFc band was larger than the theoretical molecular weight of the $\mathrm{RBD}$ of the $\mathrm{S}$ protein $(51 \mathrm{kD})$. This change in the molecular weight of S protein of SARS-Co was also shown in a previous study (Yuan et al., 2017). The $A_{450}$ boosted with the increase in the concentration of RBD-mFc expressed by our method described above in the interaction assay of the RBD$\mathrm{mFc}$ with human ACE2 (Figure S1D). In our study, the median age was 48 years (ranging from 18 years to 82 years), and $34.0 \%$ of the patients were male. To investigate the efficacy of the ELISA method, samples from 47 COVID-19 participants who were nucleic acid-positive (Table S1) and 300 healthy controls were analyzed. The 47 positive serum samples produced a median $A_{450}$ of 1.078 for rN-based $\operatorname{IgG}$, which was much higher than it was in all healthy controls (median $A_{450}$ of 0.036$)(t=42.20, P<0.0001)$ (Figure 1A). The mean $A_{450}$ value (rS-based IgG) for the serum samples obtained from the COVID-19 patients was 0.405 (range 0.032 to 1.029 ), and the normal controls had a median $A_{450}$ value of $0.079(t=17.83, P<0.0001)$ (Figure 1B). These results suggest that the ELISA was able to detect the COVID-19 IgG antibody in both rN-based IgG and rS-based IgG because all the nucleic acid-positive results detection had higher values than that in the normal controls. However, the range of $A_{450}$ value (from 0.032 to 0.402 ) in rS-based IgG testing results involve not only positive cases but also control subjects, which indicated that further testing and observation should be conducted to ensure the correct conclusion. For rN-based IgM, patients had a median value of 1.453 (range 0.381 to 2.217 ). The negative controls had lower $A_{450}$ values, with a median of $0.059(t=49.36, P<0.0001)$ (Figure 1C). For rS-based IgM, the median of positive patients was 1.398 (ranging from 0.111 to 2.818), which was higher than that of the control group $(t=31.58, P<0.0001)$ (Figure 1D). The results showed that the AUC of rN-based IgG reached 0.999 $(P<0.001$, Figure $1 \mathrm{G})$. The optimal cutoff value was 0.443 , with sensitivity and specificity values of $97.9 \%$ and $99.7 \%$, respectively. According to the cutoff value, there was only one patient who had a positive nucleotide result and a negative ELSIA result. The results showed that the AUC of rSbased IgG was $0.949(P<0.001$, Figure $1 \mathrm{H})$. Its optimal cutoff value was 0.176 , with sensitivity and specificity values of $95.7 \%$ and $85.7 \%$, respectively. The sensitivity and specificity values of rN-based $\operatorname{IgM}$ were $97.9 \%$ and $99.7 \%$, respectively, with an AUC of $0.994(P<0.001$, Figure 1I). For rS-based IgM, the optimal cutoff value was 0.167 , with sensitivity and specificity values of $89.1 \%$ and $97.0 \%$, respectively $(P<0.001$, Figure $1 \mathrm{~J})$.

In chemiluminescence, the positive patients showed much higher level in IgG, compared with normal controls $(t=7.20$, $P<0.0001$ ) (Figure $1 \mathrm{E}$ ). In IgM, the statistical difference existed between the positive and negative $(t=11.40$, $P<0.0001$ ) (Figure 1F). An analysis of the ROC curve for $\mathrm{IgG}$ in chemiluminescence demonstrated an optimal cut off value of $0.199(P<0.001$, sensitivity of $95.6 \%$ and specificity of $96.6 \%$ ). The AUC was 0.996 (Figure $1 \mathrm{~K}$ ). For IgM, the results showed that the AUC reached $0.993(P<0.001$, Figure $1 \mathrm{~L})$. The optimal cutoff value was 0.230 , with sensitivity and specificity values of $97.7 \%$ and $95.2 \%$, respectively. For patients who were confirmed positive by the nucleic acid assay but had a negative chemiluminescence result, we analyzed the corresponding $A_{450}$ by the ELISA method. Two confirmed patients had a negative rN-based IgG analysis result. Their average $A_{450}$ values were 0.651 and 1.122. One confirmed patient was negative in the rS-based IgM analysis, with an $A_{450}$ value of 1.647 . The patients' $A_{450}$ values exceeded the corresponding median value of the normal controls. Therefore, the two methods could be simultaneously performed to monitor the titer of antibodies and provide more comprehensive information.

Although detection of the RNA by either PCR or sequencing is the gold standard for COVID-19 diagnosis, false negative results of the nucleotide assay have been reported due to problems related to sample collection and/or detection methods. In this study, both the ELISA and the chemiluminescence methods exhibited good consistency with nucleic acid detection. For the ELISA, rN-based IgG was seemingly more sensitive than $\mathrm{rS}$-based IgG for discriminating between the patients and the controls. The differential sensitivity of the rS-based and rN-based IgG ELISA that was demonstrated in the present report may have originated from different restricted IgG-dominated antibody responses (Leung et al., 2004). In contrast, both rN-based IgM and rS-based IgM were able to discriminate the positive patients from the controls, so it may be a good tool to use with the nucleic acid test. If a patient has a high $A_{450}$ value for IgM but has anegative nucleic acid test result, they should be followed and 

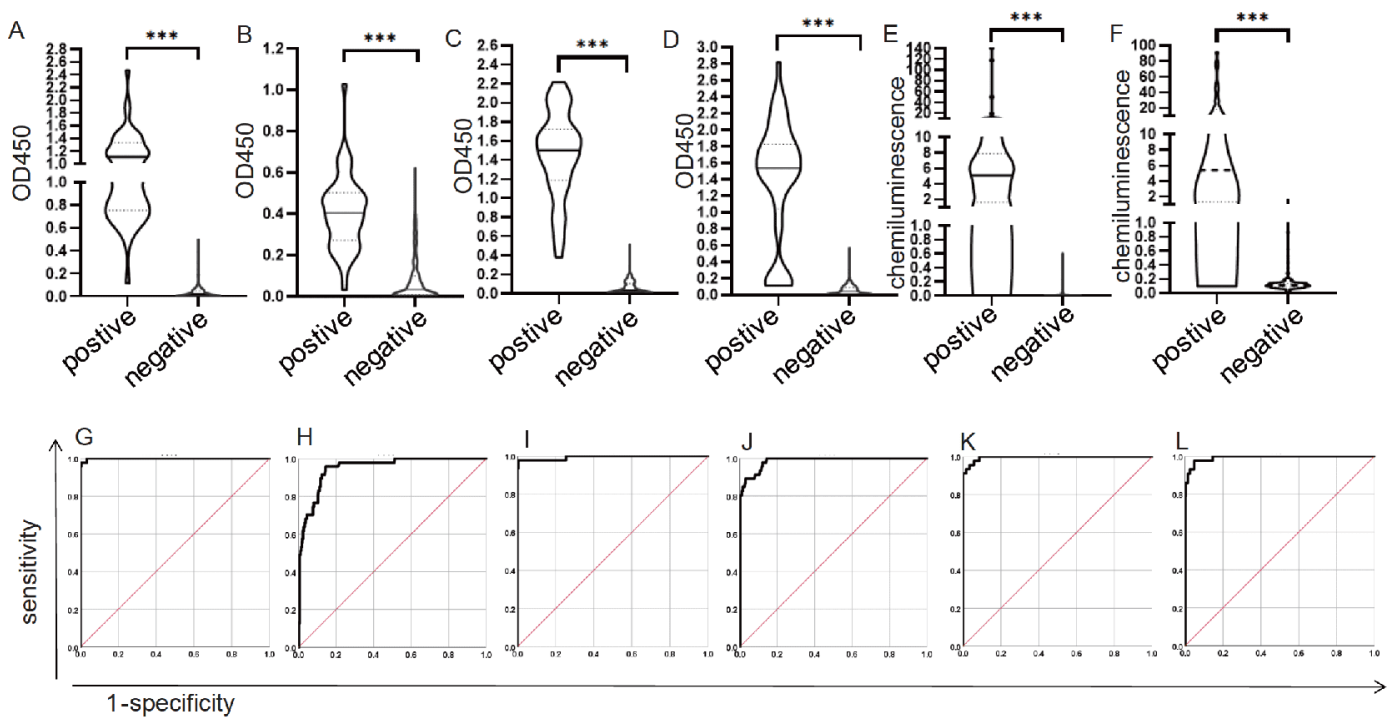

Figure 1 (Color online) Results and ROC curve of antibodies for SARS-CoV-2 detected by ELISA and chemiluminescence. A, $A_{450}$ values of the two groups (positive and negative controls) tested by rN-based IgG. B, $A_{450}$ values analyzed by rS-based IgG in different groups. C, $A_{450}$ values in two groups from rN-based IgM testing. D, For rS-based IgM analysis, different groups showed differing $A_{450}$ values $\mathrm{E}$, For IgG analysis in chemiluminescence, different groups showed differing values F, For IgM analysis in chemiluminescence, different groups showed differing values G, ROC curve for rN-based IgG. H, ROC curve analyzed by rS-based IgG. I, ROC curve from rN-based IgM testing. J, ROC curve for rS-based IgM analysis. K, The ROC curve for IgG testing in chemiluminescence. L, The ROC curve analyzed by IgM testing in chemiluminescence. $* * *, P<0.0001$.

re-tested for the virus by PCR. False-negative results from PCR may be misleading. The rS-based IgM ELISA was reported to be more sensitive than the rN-based IgM ELISA because the $\mathrm{S} 1$ protein is a transmembrane protein, which may easily stimulate the body to produce the IgM antibody, especially during early infection (Woo et al., 2005). The limitation of serum IgM and IgG for COVID-19 diagnosis may be that the time span after disease onset could affect the $A$ value, which may reduce the sensitivities of the assays. If blood samples were collected druing the early stage of the infection, they may produce false negative results. Other molecules including interferon, rheumatoid factor, non-specific IgM and so on, might cause false positive. Moreover, since the identity of the $\mathrm{N}$ protein of SARS-CoV-2 and SARS-Co reached up to $91.2 \%$, there is probably to a crossreaction between the $\mathrm{N}$ protein of SARS-CoV-2 and antibodies against other human coronaviruses. It is better to verify the detection results with the combination of assay against both the $\mathrm{N}$ protein and $\mathrm{S}$ proteins.

In conclusion, our results showed that ELISA and chemiluminescence methods to detect IgG and IgM antibodies by the recombinant $\mathrm{N}$ and $\mathrm{S}$ proteins of SARS-CoV-2 were more consistent with the nucleic acid detection assay. They not only eliminated a possible cross reaction with other coronaviruses but also avoided potential risks in the process of inactivation. The two methods are simple and cheap and require only a small amount of serum, suggesting that they could be a good prospect for wide application in seroepidemiologic monitoring and may play a valuable role in practical applications for the diagnosis and epidemic control of COVID-19 in the future.

Compliance and ethics The author(s) declare that they have no conflict of interest. This study was performed in accordance with the principles of the Helsinki Declaration of the World Medical Association. The protocol was approved by the Institutional Ethics Committee of the Sichuan Provincial People's Hospital.

Acknowledgements We thank all the participants and their families for participating in this study. This work was supported by the grants from Sichuan Science and Technology Program (2020YFS0014 and 2020YFS0558), the Chinese Academy of Medical Sciences (2019-I2M-5032) and Technology \& Science \& Technology Bureau of Chengdu (2020YF05-00060-SN and 2020-YF05-00075-SN).

\section{References}

Holshue, M.L., DeBolt, C., Lindquist, S., Lofy, K.H., Wiesman, J., Bruce, H., Spitters, C., Ericson, K., Wilkerson, S., Tural, A., et al. (2020). First case of 2019 novel coronavirus in the United States. N Engl J Med 382, 929-936.

Leung, D.T.M., Tam, F.C.H., Ma, C.H., Chan, P.K.S., Cheung, J.L.K., Niu, H., Tam, J.S.L., and Lim, P.L. (2004). Antibody response of patients with severe acute respiratory syndrome (SARS) targets the viral nucleocapsid. J INFECT DIS 190, 379-386.

Lu, R., Zhao, X., Li, J., Niu, P., Yang, B., Wu, H., Wang, W., Song, H., Huang, B., Zhu, N., et al. (2020). Genomic characterisation and epidemiology of 2019 novel coronavirus: implications for virus origins and receptor binding. Lancet 395, 565-574.

Negida, A., Fahim, N.K., and Negida, Y. (2019). Sample size calculation guide-part 4: how to calculate the sample size for a diagnostic test accuracy study based on sensitivity, specificity, and the area under the ROC curve. Adv J Emerg Med 3, e33.

Woo, P.C.Y., Lau, S.K.P., Wong, B.H.L., Tsoi, H.W., Fung, A.M.Y., Kao, R. Y.T., Chan, K.H., Peiris, J.S.M., and Yuen, K.Y. (2005). Differential sensitivities of severe acute respiratory syndrome (SARS) coronavirus spike polypeptide enzyme-linked immunosorbent assay (ELISA) and 
SARS coronavirus nucleocapsid protein ELISA for serodiagnosis of SARS coronavirus pneumonia. J Clin MicroBiol 3054-3058.

Xiao, S.Y., Wu, Y., and Liu, H. (2020). Evolving status of the 2019 novel coronavirus infection: proposal of conventional serologic assays for disease diagnosis and infection monitoring. J Med Virol 92, 464-467.

Yang, Y., Shen, C., Wang, F., Yuan, J., Li, J., Zhang, M., Wang, Z., Xing,

L., Wei, J., Peng, L., et al. (2020). Evaluating the accuracy of different respiratory specimens in the laboratory diagnosis and monitoring the viral shedding of 2019-nCoV infections. BioRxiv, doi: 10.1101/ 2020.02.11.20021493.

Yuan, Y., Cao, D., Zhang, Y., Ma, J., Qi, J., Wang, Q., Lu, G., Wu, Y., Yan, J., Shi, Y., et al. (2017). Cryo-EM structures of MERS-CoV and SARS$\mathrm{CoV}$ spike glycoproteins reveal the dynamic receptor binding domains. Nat Commun 8, 15092.

\section{SUPPORTING INFORMATION}

Figure S1 SDS-PAGE analysis and Western blot of the N protein, the RBD of the S protein, and the binding curve of the RBD-mFc to human ACE2.

Table S1 Demography and clinical classification of COVID-19 patients

The supporting information is available online at http://life.scichina.com and https://link.springer.com. The supporting materials are published as submitted, without typesetting or editing. The responsibility for scientific accuracy and content remains entirely with the authors. 